\title{
Cerebelite aguda com herniação tonsilar: relato de caso*
}

Acute cerebellitis with tonsillar herniation: a case report

\author{
Gustavo Nunes Medina Coeli ${ }^{1}$, Guilherme Carlos da Silva ${ }^{1}$, Rodrigo Ribeiro Tiengo ${ }^{2}$, José Otávio \\ Meyer Fernandes ${ }^{3}$, Walter Luis Magalhães Fernandes ${ }^{4}$, Daniel Nogueira Lacativa Lourenço ${ }^{5}$
}

\begin{abstract}
Resumo A cerebelite aguda é uma síndrome inflamatória rara frequentemente caracterizada por rápida disfunção cerebelar. Neste estudo relatamos os achados de imagem do caso de uma criança com cerebelite aguda, herniação tonsilar e hidrocefalia hipertensiva. 0 agente etiológico não foi descoberto. 0 tratamento foi conservador, com manitol e corticoide. A análise evolutiva por imagem demonstrou resolução do quadro clínico sem sequelas.

Unitermos: Cerebelite aguda; Herniação tonsilar; Ressonância magnética.
\end{abstract}

\begin{abstract}
Acute cerebellitis is a rare inflammatory syndrome frequently characterized by fast onset of cerebellar dysfunction. The present case report describes imaging findings in a child with acute cerebellitis, tonsillar herniation and hypertensive hydrocephalus. The etiologic agent has not been determined. A conservative management was adopted, with corticoid and diuretic drugs. Imaging follow-up demonstrated resolution of the clinical condition with no sequela.

Keywords: Acute cerebellitis; Tonsillar herniation; Magnetic resonance imaging.

Coeli GNM, Silva GC, Tiengo RR, Fernandes JOM, Fernandes WLM, Lourenço DNL. Cerebelite aguda com herniação tonsilar: relato de caso. Radiol Bras. 2012 Jul/Ago;45(4):244-246.
\end{abstract}

\section{INTRODUÇÃO}

A cerebelite aguda ou ataxia cerebelar aguda é uma síndrome inflamatória incomum caracterizada por uma disfunção precoce do cerebelo. Embora geralmente seja benigna e autolimitada, pode cursar com alterações fulminantes ${ }^{(\mathbf{1})}$.

É considerada uma das principais causas de disfunção cerebelar aguda na infância e sua etiologia pode ser infecciosa, usualmente viral, pós-infecciosa ou pós-vacinal $^{(\mathbf{1})}$. A origem viral pós-varicela é a mais relatada, ocorrendo em $25 \%$ dos pacientes infantes acometidos ${ }^{(2)}$.

* Trabalho realizado no Hospital Escola de Itajubá, Itajubá, MG, Brasil.

1. Médicos Residentes em Radiologia e Diagnóstico por Imagem do Hospital Escola de Itajubá, Itajubá, MG, Brasil.

2. Médico Radiologista, Membro do Colégio Brasileiro de Radiologia e Diagnóstico por Imagem (CBR), Médico Assistente do Hospital Escola de Itajubá, Itajubá, MG, Brasil.

3. Médico Neurologista do Hospital Escola de Itajubá, Professor Titular da Disciplina de Neurologia Clínica da Faculdade de Medicina de Itajubá (FMIt), Neurorradiologista da Clínica Sul Mineira Tomosul e da Clínica Magsul, Itajubá, MG, Brasil.

4. Neuropediatra do Ambulatório de Distúrbio do Aprendizado da Universidade Estadual de Campinas (Unicamp), Campinas, SP Neurorradiologista da Clínica Magsul, Pouso Alegre, MG, Brasil.

5. Médico Radiologista do Hospital Escola de Itajubá, Itajubá, MG, Brasil.

Endereço para correspondência: Dr. Gustavo Nunes Medina Coeli. Rua Oliveira Vieira de Souza, 456, Centro. Prata, MG, Brasil, 38140-970. E-mail: gustavonmc@yahoo.com.br

Recebido para publicação em 8/1/2012. Aceito, após revisão, em 5/4/2012.
O quadro clínico geralmente é variável, com febre, movimentos oculares espontâneos anormais, tremor, ataxia de tronco e membros, disartria, cefaleia, náuseas, vômitos e alteração de consciência. Menos frequentemente podem ocorrer sinais de irritação meníngea e crises convulsivas ${ }^{(\mathbf{1 , 3})}$. Complicações graves como hipertensão intracraniana, hidrocefalia obstrutiva, herniação das tonsilas e compressão de tronco cerebral são menos comuns ${ }^{(4)}$.

O diagnóstico de cerebelite aguda pode ser difícil na vigência de sinais e sintomas sutis de comprometimento cerebelar e análise do líquido cefalorraquiano normal, sendo as imagens obtidas pelo exame de ressonância magnética (RM) valiosas no diagnóstico precoce. São raros os casos documentados na literatura com imagens de RM, notadamente demonstrando as alterações evolutivas ${ }^{(\mathbf{1}, \mathbf{4 , 5})}$.

O objetivo deste trabalho é demonstrar um caso clínico de cerebelite aguda grave com evolução favorável devido ao diagnóstico e tratamento precoces.

\section{RELATO DO CASO}

Paciente do sexo feminino, 12 anos de idade, com quadro clínico de cefaleia, vômitos e inapetência há três dias, e relato de internação recente por quadro de desidratação pós-gastroenterite.

Realizou-se tomografia do crânio, que evidenciou dilatação do sistema ventricular supratentorial e apagamento difuso de sulcos e fissuras corticais.

A RM do encéfalo demonstrou áreas de hipersinal em T2 e FLAIR nos hemisférios cerebelares, associadas a efeito expansivo com apagamento dos sulcos e herniação tonsilar, além de leve impregnação leptomeníngea, compatível com cerebelite aguda (Figura 1).

Foi iniciado o tratamento com manitol e corticoide, continuado até a alta hopitalar. Decorridos três dias do início do tratamento, a paciente encontrava-se assintomática. As tomografias de controle demonstraram evolução favorável, com redução do edema e diminuição da hidrocefalia, mantendo apenas pequenas áreas hipoatenuantes nos hemisférios cerebelares. O último estudo tomográfico, feito no décimo dia de internação, encontrava-se normal.

No seguimento da paciente foram realizadas RMs, uma no $17^{\circ}$ dia (Figura $2 \mathrm{~A}$ ) e outra após 75 dias da internação (Figuras 2B e 2C). Houve boa evolução clínica e dos achados de imagem, com redução gradativa das áreas de alteração de sinal, do edema e resolução da herniação tonsilar. 




Figura 1. A: Imagem sagital ponderada em T1 demonstrando a herniação tonsilar. B: Imagem axial FLAIR com hipersinal nos hemisférios cerebelares associado a efeito expansivo com compressão sobre a margem inferior do quarto ventrículo. C: Imagem axial ponderada em T1 demonstrando tênue impregnação pelo meio de contraste paramagnético.



Figura 2. A: Imagem axial FLAIR demonstrando discreta redução das áreas de hipersinal, exame do $17^{\circ}$ dia de internação. B,C: Último controle - imagem sagital ponderada em T1 sem alterações significativas e imagem axial FLAIR demonstrando mínimo hipersinal nos hemisférios cerebelares.

\section{DISCUSSÃO}

O diagnóstico diferencial de síndromes cerebelares se faz entre processos inflamatórios/infecciosos, tumores, traumas, acidente vascular e doenças desmielinizantes. No presente relato o agente etiológico da cerebelite não foi isolado, porém a paciente apresentou história pregressa de gastroenterite. A varicela é o principal patógeno, mas quando há diarreia grave precedendo o caso, o rotavírus deve ser lembrado ${ }^{(6,7)}$.

A doença geralmente tem curso benigno e autolimitado, entretanto, quando complicada por hidrocefalia, hipertensão intracraniana e herniação tonsilar tem pior prog- nóstico. A paciente em questão apresentou tais alterações, mas com recuperação completa após tratamento clínico. O liquor apresentava pleocitose, com proteínas e glicose normais.

$\mathrm{Na}$ cerebelite o estudo tomográfico pode ser normal; já nos casos mais graves, quando houver efeito de massa, serão visualizadas alterações compatíveis com hidrocefalia obstrutiva, caracterizadas por compressão sobre o quarto ventrículo, dilatação do sistema ventricular supratentorial e apagamento difuso de sulcos e fissuras corticais ${ }^{(\mathbf{8})}$.

O estudo preconizado na avaliação do paciente com suspeita clínica de cerebelite aguda é a RM do encéfalo ${ }^{(\mathbf{1})}$. Nesse estudo, a análise da fossa posterior traz maiores detalhes que a tomografia, eliminando os artefatos gerados pelo arcabouço ósseo, facilitando a identificação e diferenciação do córtex e da substância branca dos hemisférios cerebelares. As alterações de sinal são características e juntamente com os achados clínicos fecham o diagnóstico.

Nas sequências ponderadas em T1 os hemisférios cerebelares apresentam-se com baixo sinal simétrico do córtex e nas ponderadas em T2 e FLAIR apresentam-se com hipersinal ${ }^{(\mathbf{1 , 4 , 5})}$. A difusão pode diferenciar entre edema vasogênico e citotóxico ${ }^{(6,7)}$. Com o uso do agente paramagnético as le- 
sões corticais e leptomeninges adjacentes podem sofrer impregnação anômala ${ }^{(\mathbf{1}, \mathbf{8})}$. Além disso, por ser um estudo multiplanar, pode-se avaliar o efeito de massa gerado pela inflamação do cerebelo sobre o quarto ventrículo, determinando o grau de hidrocefalia e herniação tonsilar.

As alterações de sinal nos hemisférios cerebelares nas sequências ponderadas em T2 e FLAIR na RM, como as encontradas nesta paciente, são altamente sugestivas, porém não patognomônicas. As RMs realizadas não apresentaram restrição à difusão, nem alterações do sinal no corpo caloso, o que pode estar relacionado ao tratamento precoce ou à janela entre os exames. O acompanhamento clínico e dos achados de imagem documentaram a evolução da doença e sua resolução gradativa, com redução do edema, das áreas de hipersinal e regressão da herniação tonsilar e da hidrocefalia.

O tratamento deve ser iniciado o mais precocemente possível e será realizado dependendo do agente etiológico. Nos casos de etiologia indeterminada, havendo complicações como hidrocefalia e herniação tonsilar, é mandatório o uso de manitol e corticoide ${ }^{(9)}$.

\section{REFERÊNCIAS}

1. De Bruecker Y, Claus F, Demaerel P, et al. MRI findings in acute cerebellitis. Eur Radiol. 2004;14: 1478-83.

2. Connolly AM, Dodson WE, Prensky AL, et al. Course and outcome of acute cerebellar ataxia. Ann Neurol. 1994;35:673-9.

3. Sawaishi Y, Takahashi I, Hirayama Y, et al. Acute cerebellitis caused by Coxiella burnetti. Ann Neurol. 1999;45:124-7.
4. Jabbour P, Samaha E, Abi Lahoud G, et al. Hemicerebellitis mimicking a tumor on MRI. Childs Nerv Syst. 2003;19:122-5.

5. Horowitz MB, Pang D, Hirsch W. Acute cerebellitis: case report and review. Pediatr Neurosurg. 1991-1992;17:142-5.

6. Takanashi J, Miyamoto T, Ando N, et al. Clinical and radiological features of rotavirus cerebellitis. AJNR Am J Neuroradiol. 2010;31:1591-5.

7. Kobayashi S, Negishi Y, Ando N, et al. Two patients with acute rotavirus encephalitis associated with cerebellar signs and symptoms. Eur J Pediatr. 2010;169:1287-91.

8. Kato Z, Shimozawa N, Kokuzawa J, et al. Magnetic resonance imaging of acute cerebellar ataxia: report of a case with gadolinium enhancement and review of the literature. Acta Pediatr Jpn. 1998;40: $138-42$.

9. Göhlich-Ratmann G, Wallot M, Baethmann M, et al. Acute cerebellitis with near-fatal cerebellar swelling and benign outcome under conservative treatment with high dose steroids. Eur J Paediatr Neurol. 1998;2:157-62. 